\title{
SIINTOMAS RESPIRATORIOS ASOCIADOS AL HUMO DEL ELECTROCAUTERIO: REVIIIÓN SISTEMÁTICA DE LA LITERATURA
}

Mariana Alejandra Palomino Castro*, Manuela Usma Zapata**, Geraldine Triana Bermeo***, Angélica María Blanco Vanegas****, Germán Oved Acevedo Osorio*****, Ángela María Rincón Hurtado******

\section{Resumen}

Introducción: la exposición a la inhalación de sustancias tóxicas por medio del uso del electrocauterio en procedimientos quirúrgicos, puede ser la causa de síntomas respiratorios en instrumentadores quirúrgicos, ya que las partículas de humo generadas por este equipo médico son altamente irritantes debido a su composición química. Metodología: búsqueda sistemática de la literatura con metodología Scoping Review. Se realizó una búsqueda bibliográfica en las bases de datos e índices: Pubmed, Science Direct, Scopus, Proquest, Scielo, Scholar Google. Resultados: se recuperaron un total de ciento veintiún artículos, de estos noventa y cuatro se excluyeron del análisis posterior debido a la falta de datos apropiados; los veintisiete estudios de texto completo restantes publicados entre 2015 y 2020 cumplieron con los criterios de inclusión y exclusión planteados en el estudio, los cuales se excluyeron en la revisión. Hubo ocho estudios relacionados con los componentes del humo quirúrgico y los síntomas o patologías respiratorias, de los cuales tres son estudios de revisión sistemática,

* Terapeuta Respiratorio. Fundación Universitaria del Área Andina. Pereira, Colombia. Correo: mpalomino6@estudiantes.areandina.edu.co. Orcid: ttps://orcid.org/00000002-4639-248X Caldas, Colombia.

* Estudiante de Instrumentación Quirúrgica. Fundación Universitaria del Área Andina. Pereira, Colombia. Correo: musma2@estudiantes.areandina.edu.co. Orcid: https:// orcid.org/0000-0003-0435-0705 Risaralda, Colombia.

*** Estudiante de Instrumentación Quirúrgica. Fundación Universitaria del Área Andina. Pereira, Colombia. Correo: gtriana3@estudiantes.areandina.edu.co. Orcid: https:// orcid.org/0000-0002-1762-2901 Risaralda, Colombia.

**** Terapeuta Respiratorio. Magíster en Salud Pública y Desarrollo Social. Fundación Universitaria del Área Andina. Pereira, Colombia. Correo electrónico: ablanco2@ areandina.edu.co. Orcid: https://orcid.org/0000-0001-7021-1944 (autor para correspondencia).

***** Instrumentador Quirúrgico. Magíster en Salud Pública y Desarrollo Social. Fundación Universitaria del Área Andina. Pereira, Colombia.

****** Phd. Ciencias de la Salud Pública, Mgs en Epidemiología, Grupo los Asépticos. 
un estudio cualitativo, un estudio descriptivo, un estudio de revisión de literatura y dos no reportaban el tipo de estudio. Conclusiones: los riesgos ante la inhalación del humo del electrocauterio pueden desencadenar patologías respiratorias crónicas como el cáncer pulmonar; por tanto, se deben generar estrategias para la prevención de la enfermedad como el uso adecuado de los elementos de protección personal y la implementación de sistemas de evacuación. 


\section{Introducción}

El electrocauterio es un dispositivo médico que genera corriente eléctrica, teniendo como objetivo la producción de calor. Esto funciona por medio de una tecnología de alta frecuencia y con un voltaje y frecuencia determinados para realizar hemostasia en pequeños vasos sanguíneos e incluso cortar y coagular tejidos, y así brindar buenos resultados $y$ un menor tiempo de exposición quirúrgica. Estos dispositivos pueden llegar a alcanzar temperaturas superiores a los $200^{\circ} \mathrm{C}$. Por este motivo, pueden utilizarse en diferentes especialidades y tienen distintas funciones, como realizar una coagulación diseminada (1).

El humo producido por el uso de la electrocauterización está formado por compuestos químicos gaseosos (fase gaseosa) y por componentes de partículas (fase particulada) que pueden desencadenar efectos nocivos, locales o sistémicos, reversibles o irreversibles en personas que usan este equipo. Con respecto a la composición química, puede contener hidrocarburos aromáticos policíclicos (HAP), compuestos orgánicos volátiles (COV), monóxido de carbono (CO), entre otros. Estos productos químicos pueden desencadenar mutaciones genéticas y cáncer en el cuerpo humano. Además de la mutación y el cáncer, las partículas en humo de la electrocauterización se pueden inhalar y retener en el tracto respiratorio de los trabajadores, causando diversos signos y síntomas respiratorios, incluida la sensación de cuerpo extraño en la garganta, náuseas, ardor faríngeo y congestión nasal. También puede causar dolor de cabeza e irritación ocular (2).
La exposición a la inhalación de sustancias tóxicas por medio del uso del electrocauterio en procedimientos quirúrgicos, puede ser la causa de síntomas respiratorios en instrumentadores quirúrgicos, puesto que las partículas de humo generadas por este equipo médico son altamente irritantes debido a su composición química. Este equipo tiene diferentes variables que se aplican según el tipo de tejido cauterizado, energía bipolar o monopolar, dispositivo utilizado, duración de la intervención, estado inmunológico en el que se encuentre el paciente, enfermedad a la que se le va a realizar, entre otras. Hay estudios in vitro que nos han ayudado a encontrar diferentes evidencias en las que dice que el humo del electrocauterio podría tener un efecto mutagénico sobre el epitelio respiratorio. A esto le agregamos que los agentes químicos resultantes de la pirólisis de los tejidos tienen un olor no muy particular, el cual, cuando lo sumamos a los componentes del mismo, puede ocasionar inmediatamente malestar y síntomas respiratorios diversos, como ardor faríngeo, tos e irritación $(3,4)$.

Se debe tener en cuenta la función de la mascarilla quirúrgica usada en las salas de cirugía, pues estas se utilizan para generar una barrera física y así proteger a los empleados de riesgos como salpicaduras de fluidos corporales o sangre. Pero según la Administración de Seguridad y Salud Ocupacional (OSHA, por sus siglas en inglés) de los Estados Unidos, no se encuentran certificadas para prevenir la inhalación de dichos contaminantes transportados por el aire. Por esta razón, la OSHA recomienda el uso de mascarillas y respiradores certifica- 
dos por el Instituto Nacional para la Salud y Seguridad Ocupacional (NIOSH, por sus siglas en inglés) (5).

\section{Metodología}

Búsqueda sistemática de la literatura mediante la metodología de Scoping Review. Se realizó una búsqueda bibliográfica en las bases de datos e índices: Pubmed, Science Direct, Scopus, Proquest, Scielo, Scholar Google. Se seleccionaron artículos originales publicados entre 2015-2020 con la intención de explorar la producción científica generada en los años más recientes sobre el tema, escritas en idioma inglés y español; previa revisión del título y resumen se procedió a recuperar el texto completo (véase diagrama de selección de los artículos de revisión). Para la extracción y síntesis del contenido de los artículos incluidos, se diseñó una hoja de Excel con las siguientes variables: autores, título, año de publicación, país y revista científica; objetivo del estudio, materiales y métodos, población y muestreo, resultados reportados. La calidad de los datos recogidos fue verificada por dos integrantes del equipo investigador a través de reuniones de acuerdo y consenso entre ellos. Teniendo en cuenta los requerimientos éticos para la investigación documental, se protegen los derechos de autor, citando apropiadamente el documento.

Se utilizaron los términos del Medical Subject Headings [MeSH]. Especialmente, se realizó la siguiente combinación: "Health Personnel" AND "Smoke" AND "Electrosurgery" AND "Lung Diseases", en cada una de las bases de datos e índices seleccionados en el estudio.

\section{Resultados}

Se recuperaron 121 artículos, de estos 94 se excluyeron del análisis posterior debido a la falta de datos apropiados, los 27 estudios de texto completo restantes cumplieron con los criterios de inclusión y exclusión planteados en el estudio, los cuales se excluyeron en la revisión.

Los estudios incluidos fueron publicados entre 2015 y 2020, principalmente en América del Sur, América Latina y Europa. Hubo ocho estudios relacionados con los componentes del humo quirúrgico y los síntomas o patologías respiratorias, de los cuales tres eran estudios de revisión sistemática, un estudio cualitativo, un estudio descriptivo, un estudio de revisión de literatura y dos no reportaban el tipo de estudio.

De los 27 estudios incluidos en esta revisión, hubo diecinueve estudios que no reportaron el tiempo de exposición al humo del electrocauterio, trece no reportaron el uso de elementos de protección personal, catorce informaron del uso de N95 o mascarilla quirúrgica, ocho reportaron el tiempo de exposición al humo quirúrgico y solo tres estudios señalaron la relación entre el tiempo de exposición y el uso de elementos de protección personal.

Con relación a los resultados asociados con el tiempo de exposición al humo del electrocauterio y el uso de elementos de protección personal, Vieira et al. (6), en el 2017, realizaron un estudio de campo, exploratorio y transversal con abordaje cuantitativo en el equipo intraoperatorio de un centro quirúrgico en el norte de 
Paraná, Brasil, donde tuvieron como resultado que, en promedio, la exposición al humo del electrocauterio en los procedimientos quirúrgicos fue de 3,6 minutos, y el uso de mascarilla quirúrgica se dio en el $90 \%$ de los trabajadores, pero ningún trabajador perteneciente al equipo intraoperatorio, utilizó algún tipo de máscara respiratoria, como la N95.

En el 2015, Lindsey et al. (7) efectuaron una revisión sistemática donde reportaron que la mayoría de las máscaras quirúrgicas solo filtran partículas de aproximadamente $0,5 \mathrm{~mm}$ de tamaño; sin embargo, la mayoría de las partículas de las plumas son ultrafinas y mucho más pequeñas. Por otro lado, Yi Liu et al. (8), en el 2019, al hacer un estudio de revisión, determinaron que para evitar la lesión por inhalación del humo quirúrgico, se debe usar protección respiratoria, como una mascarilla quirúrgica básica, máscara de alta filtración, o un respirador $\mathrm{N} 95$.

Navarro et al. (9), en el 2016, realizaron un estudio con el objetivo de demostrar que la exposición al humo producto de la electrocoagulación, origina cambios en la mucosa nasal en médicos en formación de un hospital público en México, donde obtuvieron como resultado que el mayor tiempo de exposición a dicho humo se da en las especialidades del servicio de neurocirugía y de cirugía general, los cuales presentaban un tiempo de exposición de 16 a 22 minutos, aproximadamente, generando cambios en la mucosa nasal.

En cambio, Atar et al. (10), en el 2017, en Turquía realizaron un estudio experi- mental con 16 ratas albinas Wistar adultas y sanas, expuestas al humo durante $60 \mathrm{~min} /$ día durante cuatro semanas, y estas mostraron un aumento de la inflamación del tejido debido a la irritación por el humo.

\section{Discusión}

Durante muchos años, ha habido interés en determinar y definir exactamente qué peligro representa el humo quirúrgico, desde determinar los riesgos de inhalar dicho humo, así como establecer los peligros potenciales acumulables. Aunque existe una gran cantidad de información y recomendaciones, no se ha establecido un vínculo específico entre la exposición al humo quirúrgico y los efectos adversos para la salud del personal perioperatorio. No hay regulaciones obligatorias para Colombia y el mundo que indiquen que se debe evacuar el humo quirúrgico, pero los estándares de las organizaciones profesionales indican claramente que existe un peligro potencial si el personal inhala continuamente sustancias presentes en el humo quirúrgico (11).

Hay evidencias crecientes que sugieren que las partículas de aproximadamente $5 \mu \mathrm{m}$ o más se depositan en las paredes de la nariz, la faringe, la tráquea y los bronquios; mientras que las de menos de 2 micrómetros se depositan en los bronquiolos y los alvéolos (12). La carga química del cauterio de un gramo de tejido es comparable a la derivada de seis cigarrillos. Se ha aislado el ADN viral del VIH y del VPH, y tanto el Staphylococcus como la Neisseria se cultivaron a partir de humo quirúrgico (13). Es de esta manera que distintos estudios evi- 
dencian que las mascarillas quirúrgicas estándar no ofrecen protección; mientras que los dispositivos portátiles de evacuación son la mejor medida de reducción de riesgos.

Es considerable que las instituciones de salud contemplen distintos mecanismos para eliminar un peligro controlable como el humo, el cual puede ayudar a minimizar los costos de salud y mejorar la salud del personal quirúrgico. Además, estos esfuerzos permiten controlar este riesgo ambiental en el ámbito laboral.

Durante los procedimientos quirúrgicos de cirugía abierta, hay distintas formas para que el personal de quirófano evite el humo quirúrgico, como moviéndose o alejándose de grandes columnas de humo y evitando así la inhalación. Pueden utilizarse máscaras de filtro de mayor calidad o doble enmascaramiento. Es recomendable utilizar dispositivos de succión como de evacuación de humo simple cerca de la cuchilla de la electrocauterización $(2-3 \mathrm{~cm})$ cuando se produce el humo. Si se coloca demasiado lejos, solo se evacuará el $50 \%$ del humo de un sistema de evacuación el cual debería ser muy eficiente; además, debe ser un dispositivo de succión que no interfiera con las actividades o maniobras intraoperatorias, con una fuente de vacío y filtrado lo suficientemente para convertir el ambiente en un área segura para el equipo quirúrgico.

\section{Agradecimientos}

Queremos expresar los más profundos agradecimientos a nuestras asesoras temáticas Angélica María Blanco Va- negas, Ángela María Rincón Hurtado, a nuestro asesor metodológico Germán Oved Acevedo Osorio, por su compromiso y dedicación en la realización de dicho estudio y a la Fundación Universitaria del Área Andina por contribuir en nuestra formación.

\section{Referencias}

1. International $H$ and $B$. ¿Por qué el electrocauterio es una herramienta que no puede faltar en una cirugía? [Internet]. Health and Beauty International. 2017. https:// www.healthandbeautyint.com/2017/06/05/ por-que-el-electrocauterio-es-una-herramienta-que-no-puede-faltar-en-una-cirugia/

2. Tramontini CCGCcC et al. Composition of the electrocautery smoke: Integrative literature review. J Sch Nurs usp [Internet]. 2016. https://pubmed.ncbi.nlm.nih. gov/27007432/

3. Navarro-Meza MC, González-Baltazar $\mathrm{R}$, Aldrete-Rodríguez MG, Carmona-Navarro DE, López-Cardona MG. Síntomas respiratorios causados por el uso del electrocauterio en médicos en formación quirúrgica de un hospital de México. Rev Peru Med Exp Salud Publica. 2013; 30(1):41-44.

4. Carbajo Rodríguez $\mathrm{H}$, Aguayo JL, Soria VL. El humo quirúrgico: riesgos y medidas preventivas. Cir Esp. 2009; 85(5):274-279.

5. Ministerio de Salud y Protección Social. Tómate la vida con un segundo aire. Estrategia para la prevención y control de las enfermedades respiratorias crónicas. OpsOms [Internet]. 2016; 2:1-91. https://www. minsalud.gov.co/sites/rid/Lists/BibliotecaDigital/RIDE/VS/PP/ENT/estrategia-enfermedades-respiratorias-cronicas-2017.pdf

6. Claudio CV, Ribeiro RP, Martins JT, Marziale MHP, Solci MC, Dalmas JC. Hidrocarbonetos policíclicos aromáticos produzidos pela fumaça do eletrocautério e uso de equipamentos de proteção individual. Rev Lat Am Enfermagem. 2017; 25. 
7. Lindsey C, Hutchinson M, Mellor G. The nature and hazards of diathermy plumes: $A$ review.AORN J [Internet]. 2015; 101(4):428442. https://www.mendeley.com/catalogue/ ccf48e1d-2ea4-31b4-85a5-ea85a67b$\mathrm{fcfe} /$ ?utm_source=desktop\&utm_medium=1.19.4\&utm_campaign=open_ catalog\&userDocumentld=\%7Bb0c081db-14f2-4651-b4d8-2a8ec2a52beb\%7D

8. Liu Y, Song Y, Hu X, Yan L, Zhu X. Awareness of surgical smoke hazards and enhancement of surgical smoke prevention among the gynecologists [Internet]. Vol. 10, Journal of Cancer. 2019; 27882799. https://www.mendeley.com/catalogue/8f63eb8a-7304-3f1e-9478-3b35efaf79ea/?utm_source=desktop\&utm_medium $=1.19 . \overline{4} \& u t m \_c a m p a i g n=o p e n \_c a-$ talog \&userDocumentld = \% 7B 49bdf077-b87c-49fe-ada8-17de997dfc67\%7D

9. Navarro MC, González R, Aldrete MG, Carmona DE. Cambios en la mucosa nasal de los médicos por exposición al humo por electrocoagulación. Rev Fac Nac Salud Pública. 2016; 34(2).

10. Atar $Y$, Salturk Z, Kumral TL, Uyar Y, Cakir C, Sunnetci G, et al. Effects of smoke generated by electrocautery on the larynx [Internet]. Vol. 31, Journal of Voice. Elsevier Inc. 2016; 380. e7-380.e9. https://www.mendeley.com/ catalogue/cab8ab2d-c3ee-345c-8501-bd$879 \mathrm{fe} 5 \mathrm{da} 8 \mathrm{f} /$ ? utm_source=desktop\&utm_medium=1.19.4\&utm_campaign=ōpen_catalog\&userDocumentl$\mathrm{d}=\% 7 \mathrm{~B} 04 \mathrm{c} 28191-70$ ca-4190-be9a-01aeb5f9a448\%7D

11. Brenda C Ulmer. The hazards of surgical smoke [Internet]. National library of medice. 2017. https://pubmed.ncbi.nlm. nih.gov/18461735/

12. Kae Okoshi, Katsutoshi Kobayashi, Koichi Kinoshita, Yasuko Tomizawa SH \& YS. Health risks associated with exposure to surgical smoke for surgeons and operation room personnel. the egyptian journal of otolaryngoly. 2014.
13. S Addley DQAAH. Prevalence of presenteeism and its associated factors among nurses in a teaching hospital in $\mathrm{Ni}-$ geria. 2016; 321. 